\title{
Brachial Arterial Pressure Monitoring during Cardiac Surgery Rarely Causes Complications
}

\author{
Asha Singh, M.D., Bobby Bahadorani, D.O., Brett J. Wakefield, M.D., Natalya Makarova, M.S., \\ Priya A. Kumar, M.D., Michael Zhen-Yu Tong, M.D., M.B.A., Daniel I. Sessler, M.D., \\ Andra E. Duncan, M.D., M.S.
}

\begin{abstract}
Background: Brachial arterial catheters better estimate aortic pressure than radial arterial catheters but are used infrequently because complications in a major artery without collateral flow are potentially serious. However, the extent to which brachial artery cannulation promotes complications remains unknown. The authors thus evaluated a large cohort of cardiac surgical patients to estimate the incidence of related serious complications.

Methods: The institutional Society of Thoracic Surgeons Adult Cardiac Surgery Database and Perioperative Health Documentation System Registry of the Cleveland Clinic were used to identify patients who had brachial artery cannulation between 2007 and 2015. Complications within 6 months after surgery were identified by International Classification of Diseases, Ninth Revision diagnostic and procedural codes, Current Procedural Terminology procedure codes, and Society of Thoracic Surgeons variables. The authors reviewed electronic medical records to confirm that putative complications were related plausibly to brachial arterial catheterization. Complications were categorized as (1) vascular, (2) peripheral nerve injury, or (3) infection. The authors evaluated associations between brachial arterial complications and patient comorbidities and between complications and in-hospital mortality and duration of hospitalization.

Results: Among 21,597 qualifying patients, 777 had vascular or nerve injuries or local infections, but only 41 (incidence $0.19 \%$ [ $95 \%$ CI, 0.14 to $0.26 \%$ ]) were potentially consequent to brachial arterial cannulation. Vascular complications occurred in 33 patients $(0.15 \%$ [0.10 to $0.23 \%])$. Definitely or possibly related infection occurred in $8(0.04 \%$ [0.02 to $0.08 \%]$ ]) patients. There were no plausibly related neurologic complications. Peripheral arterial disease was associated with increased risk of complications. Brachial catheter complications were associated with prolonged hospitalization and in-hospital mortality.

Conclusions: Brachial artery cannulation for hemodynamic monitoring during cardiac surgery rarely causes complications. (Anesthesiology 2017; 126:1065-76)
\end{abstract}

$\mathbf{I}$ NTRAARTERIAL pressure monitoring is routine for patients having cardiac surgery; however, the ideal site for arterial cannulation that best reflects central aortic pressure while minimizing line-associated complications is unclear. Radial arterial cannulation often is preferred because collateral circulation from the ulnar artery to the hand reduces the risk of ischemic injury. ${ }^{1}$ However, radial arterial pressure often exaggerates central aortic pressures because of decreased arterial elasticity, amplification of harmonic resonance, and the water hammer effect, which describes a bounding and forceful pulse caused by the propagation of a pressure wave throughout the vasculature. ${ }^{2}$ Furthermore, hemodilution or radial artery vasospasm during critical periods of cardiac surgery can cause radial arterial pressure to underestimate central aortic pressure. ${ }^{3,4}$ Inaccurate pressure measurements even for brief periods of time may promote inappropriate hemodynamic management and possibly increase postoperative morbidity and mortality. ${ }^{5}$

\section{What We Already Know about This Topic}

- Brachial arterial catheters better estimate aortic pressure than radial arterial catheters but are used infrequently because of risk of complications in a major artery without collateral flow are potentially serious

- The present study evaluated a large cohort of cardiac surgical patients to estimate the incidence of brachial artery catheter complications

\section{What This Article Tells Us That Is New}

- Brachial artery cannulation for hemodynamic monitoring during cardiac surgery rarely causes complications

Brachial arterial monitoring, in contrast, closely reflects central aortic pressure even during complex cardiac surgical procedures with prolonged cardiopulmonary bypass support. ${ }^{3}$ Brachial arterial pressure may thus better guide patient care and clinical management than radial arterial pressure

Submitted for publication September 27, 2016. Accepted for publication February 28, 2017. From the Anesthesiology Institute, Cleveland Clinic, Cleveland, Ohio (A.S., B.B., B.J.W.); Departments of Quantitative Health Sciences and Outcomes ReseArCH, Cleveland Clinic, Cleveland, Ohio (N.M.); Department of Anesthesiology, University of North Carolina, Chapel Hill, North Carolina (P.A.K.); Outcomes Research Consortium, Cleveland, Ohio (P.A.K.); Department of Thoracic and Cardiovascular Surgery, Cleveland Clinic, Cleveland, Ohio (M.Z.-Y.T.); and Departments of Outcomes Research (D.I.S., A.E.D.) and Cardiothoracic Anesthesiology (A.E.D.), Anesthesiology Institute, Cleveland Clinic, Cleveland, Ohio. 
monitoring. ${ }^{6}$ However, brachial artery catheterization often is avoided because the brachial artery is the major blood supply to the forearm and hand. Thrombotic and ischemic complications related to brachial artery cannulation can therefore critically compromise blood flow. Other potentially serious complications include infections and injuries to the median nerve. Complications consequent to brachial artery cannulation may be especially common in patients with diabetes and peripheral vascular disease-who are especially likely to require cardiac surgery.

The incidence of complications related to brachial artery cannulation remains essentially unknown. Two recent reports, for example, each included fewer than 150 patients undergoing cardiac surgery, ${ }^{7,8}$ making them roughly two orders-of-magnitude smaller than necessary to accurately characterize the incidence of complications, much less potential associations between comorbidities and cannulation-related complications.

We therefore evaluated vascular complications, nerve injury, and infections plausibly related to brachial arterial cannulation in patients undergoing cardiac surgery. Second, we examined whether patients with preexisting peripheral vascular disease, diabetes mellitus, carotid artery disease, coronary artery disease, or chronic kidney disease were at increased risk for complications from brachial arterial cannulation. Finally, we assessed the association between complications from brachial arterial cannulation and length of hospitalization and risk of in-hospital mortality.

\section{Materials and Methods}

With Cleveland Clinic Institutional review board (Cleveland Clinic, Cleveland, Ohio) approval and waiver of informed consent, we reviewed the electronic records of patients who underwent cardiac surgery between January 1, 2007, and March 31, 2015. Using our institutional Society of Thoracic Surgeons (STS) database and the Perioperative Health Documentation System (PHDS) registry of the Cleveland Clinic, we identified adults who had brachial arterial catheters inserted intraoperatively for hemodynamic monitoring during cardiac surgery. Patients who had previous surgery on the brachial artery or known brachial artery disease were excluded.

Cannulation of the left brachial artery for intraarterial pressure monitoring is considered standard of care for patients who present for cardiac surgery at the Cleveland Clinic. Arterial puncture is performed with a 20 -gauge intravenous catheter; the Seldinger technique (catheter-over-thewire) is then used to place a 5-inch, 20-gauge catheter for continuous arterial pressure monitoring.

Both registries were used to identify patients with potential brachial arterial catheter complications by detecting all documented vascular or nerve injury, loss of function, or infection within 6 months after the cardiac surgery. The International Classification of Diseases, Ninth Revision diagnostic and procedural codes and Current Procedural
Terminology procedure codes that were used to identify potential brachial arterial catheter complications from the PHDS are listed in appendix 1. Potential complications related to brachial arterial cannulation also were identified by a search of the institutional STS database for noncardiac reoperations, limb ischemia, postoperative anticoagulation, postoperative infections involving an arm, septicemia, and neuroparalysis.

Among patients with potential brachial arterial complications, electronic medical records were reviewed individually to characterize complications as definitely related, possibly related, or unrelated to brachial arterial line cannulation. Initial chart reviews were performed by one of three anesthesia residents (A.S., B.B., B.J.W.). All definitely and possibly related complications were cross-validated by a second anesthesiology resident to confirm that the complication appeared related. All complications and any disagreements were adjudicated by an experienced attending cardiothoracic anesthesiologist (A.E.D.).

Complications were considered definitely or possibly related to brachial artery cannulation when (1) the complication occurred at the same site and after insertion of the brachial arterial catheter; (2) the daily intensive care unit (ICU) progress note, cardiac surgery progress note, vascular medicine/surgery consult note, neurology consult note, operative reports, or infectious disease consult notes stated that the complication was consequent to the brachial arterial line; and (3) the brachial arterial catheter was removed when the complication was identified. Once complications were deemed definitely or possibly related to brachial arterial cannulation, they were categorized as a vascular (brachial arterial injury, upper extremity ischemia, compartment syndrome), neurologic (median nerve injury, paresthesia), or infectious (local or systemic infection).

Brachial arterial injuries were further categorized as thrombotic, embolic, dissection, stricture, aneurysm, or other arterial injury. If compartment syndrome was identified, the site was recorded as upper arm, forearm, wrist, or hand. We recorded whether treatments were required, including surgical arterial repair, thrombectomy, fasciotomy (upper arm, forearm, wrist, hand), anticoagulation, or amputation.

Infectious complications were categorized as cathetersite infection, cellulitis, bloodstream infection, septicemia, or other infection. When bloodstream infection or septicemia was identified, we categorized the events as definitely or possibly related to the brachial arterial catheter. Infections were considered definitely related to arterial catheterization and thus the likely source of bloodstream infection or septicemia when one of the following criteria were met: (1) the organism identified from culture of the catheter tip matched the blood culture; (2) the ICU progress note or infectious disease note described a local infection at the site of brachial arterial cannulation; (3) the ICU daily progress 
note or infectious disease consult note stated that the arterial catheter was the likely source of infection; (4) other sources of infection were considered unlikely (for example, there were no other intravascular catheters). It is routine at our institution to remove all intravascular catheters when bloodstream infection or septicemia is identified, even without direct evidence that a catheter was the source of infection. Infectious complications were thus labeled as possibly related to arterial catheterization if none of the aforementioned criteria for definitely related to brachial arterial catheterization were fulfilled. We recorded whether treatments were required, including cannulation-site debridement and antibiotic therapy.

Patient demographics, comorbidities (diabetes, peripheral vascular disease, carotid disease, coronary artery disease, chronic kidney disease), type of cardiac surgery, length of hospital stay, and in-hospital mortality also were obtained from the registries.

Carotid artery disease, which is evaluated routinely by carotid ultrasound before cardiac surgery at our institution, was defined by the STS (version 2.81) data specification as carotid stenosis $50 \%$ or greater. Diabetes and peripheral vascular disease also were defined according to the STS data specifications. Patients having coronary artery bypass grafting were considered to have coronary artery disease. Chronic kidney disease was defined as estimated glomerular filtration rate less than $30 \mathrm{ml} \cdot \mathrm{min}^{-1} \cdot 1.73 \mathrm{~m}^{-2}$ calculated via the Modification of Diet in Renal Disease estimated glomerular filtration rate formula based on serum creatinine, age, gender, and race.

\section{Statistical Analysis}

We summarized patient's demographic and medical baseline characteristics as well as procedure related details via standard univariable summary statistics as means $\pm S D$, median [first quartile, third quartile], or $\mathrm{n}(\%)$.

The incidence of complications related to the intraoperative brachial arterial line insertion, our primary outcome, was estimated along with the $95 \% \mathrm{CI}$, assessed via normal approximation theory. ${ }^{9}$ The separate incidence of brachial artery vascular complications, neurologic complications, and infection along with $98.3 \%$ (correction for multiple outcomes) normality approximated CI also was reported.

Second, we reported the association between the presence of diabetes, peripheral vascular disease, carotid artery disease, chronic kidney disease and coronary artery disease, and the risk of complications from brachial arterial cannulation with multivariable logistic regression. The association between chronic kidney disease and risk for brachial complications was assessed post hoc. We considered brachial arterial complications as the binary outcome and the listed conditions as the explanatory factors. Given the low incidence of the outcomes, we limited the adjustment of the reported associations to preselected clinically relevant cofounders, which included patient's age, body mass index, sex, race, American
Society of Anesthesiologists physical status, duration of surgery, surgery emergency status, number of simultaneous cardiac procedures, duration of cardiopulmonary bypass time, duration of myocardial ischemia, and duration of circulatory arrest. The Wald test $P$ value and the adjusted odds ratio along with $99 \%$ (correction for multiple comparisons) CI were reported for each of the baseline condition.

For the tertiary outcomes, we examined whether a complication related to brachial arterial cannulation was associated with increased length of hospitalization via multivariable linear regression model with log-transformed (to satisfy model normality assumption) length of hospitalization as an outcome and status on brachial arterial complication as an exploratory factor. The Wald test $P$ value and the adjusted ratio of geometric means along with $97.5 \%$ (correction for multiple comparisons) CI were reported. The association between in-hospital mortality and brachial arterial cannulation complications were examined via multivariable logistic regression models. We reported the Wald test $P$ value and the adjusted odds ratio along with $97.5 \%$ (correction for multiple comparisons) CI. Both associations were adjusted for history of diabetes, peripheral vascular disease, carotid disease, coronary artery disease, and all the baseline and intraoperative factors listed for the secondary analysis adjustment.

To adjust for multiple reported comparisons, the Bonferroni correction was applied for the computed CIs and tests significance levels to maintain the Type I error rate at 5\% on each primary, secondary and tertiary analysis. SAS 9.4 statistical software (SAS Institute Inc., USA) was used for all analysis.

\section{Sample Size Consideration}

We planned to use all available cases and anticipated approximately 3,000 eligible patients per year, total of 24,000 patients. With 24,000 patients, we have the following precision in estimating complication incidence (precision is described in terms of CI width): the width of $95 \% \mathrm{CI}$ ranges from 0.06 to $0.12 \%$ for incidence ranging from 0.05 to $0.2 \%$, respectively: CI width was estimated by use of exact binomial method.

\section{Results}

After review of the PHDS and STS registries, we identified 30,652 patients who had cardiac surgery between 2007 and 2015. In 6,000 patients, documentation for an arterial catheter was missing. Most of the surgeries with missing documentation occurred between 2007 and mid-2008 (and were related to design issues in the newly implemented electronic anesthesia record); these patients were therefore excluded from analysis. A total of 21,597 patients met our inclusion and exclusion criteria (fig. 1), with 777 coded as having some form of vascular injury, nerve injury, loss of function, or infection within 6 months after cardiac surgery. Manual review of the electronic medical records confirmed 


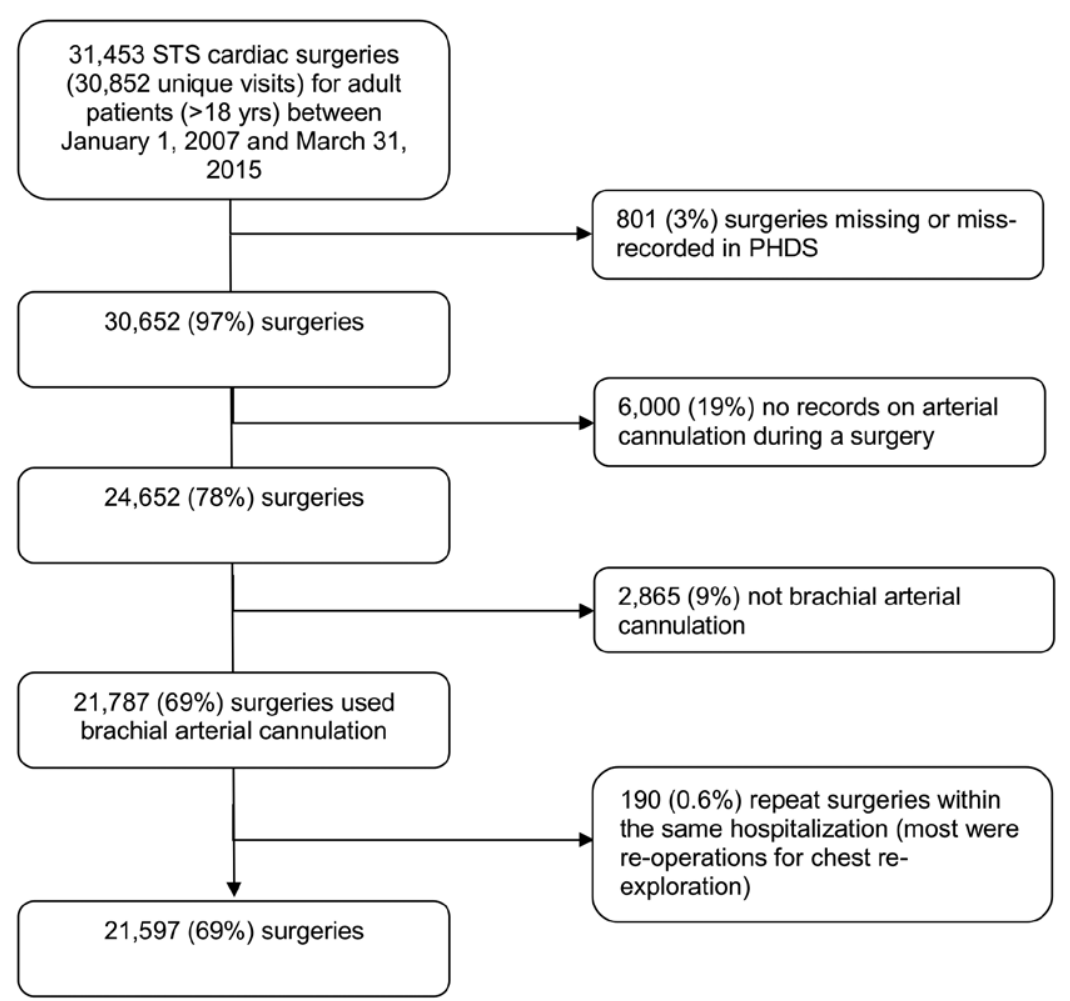

Fig. 1. Flowchart of patient selection. PHDS = Perioperative Health Documentation System; STS = Society of Thoracic Surgeons.

that 41 of 777 patients had complications that were definitely or possibly related to intraoperative brachial arterial catheter insertion. The corresponding incidence estimate $(95 \% \mathrm{CI})$ for the combination of all three complications is $0.19 \%(0.14$ to $0.26 \%)$.

Demographic and baseline characteristics of the study population and procedural details for patients with and without brachial arterial complications are shown in table 1 .

Complications related to brachial arterial cannulations occurred between the day of surgery and the 30th postoperative day with the median [first, third quartile] day of complication on postoperative day $4[2,8]$. Brachial artery vascular complications occurred in $33(0.15 \%$ [0.10 to $0.23 \%])$ patients, who had one or more of the following: upper extremity ischemia (21 patients), thrombosis (17 patients), embolism (2 patients), aneurysm (4 patients), compartment syndrome (1 patient), and other arterial injury (1 patient; table 2). No documented neurologic complications were found in any patient (estimated incidence $0.00 \%$ [0.00 to $0.03 \%])$. Infection occurred in $8(0.04 \%$ [0.02 to $0.08 \%])$ patients. Five of eight infections were catheter-related sepsis. It was not possible to determine whether the infection originated from the arterial catheter or from coexistent centralvenous catheters; thus, these complications were considered possibly related.

Treatment was required for 37 of the 41 patients who experienced complications (appendix 2). Treatment for vascular complications included repair of the brachial artery in $8(22 \%)$ patients, thrombectomy in $18(49 \%)$ patients, and fasciotomy in $1(3 \%)$ patient. Other therapeutic interventions included anticoagulation in $17(46 \%)$ patients, direct vascular compression in $3(8 \%)$ patients, Rooke ${ }^{\circledR}$ Mitts (which promote vasodilation; Osborn Medical, USA) in $3(8 \%)$, and thrombin injection in 1 patient (3\%). All 8 cases of infection were treated with systemic antibiotic therapy.

Univariable and multivariable associations between the presence of diabetes, peripheral vascular disease, carotid disease, chronic kidney disease, coronary artery disease, and the risk of complications from brachial arterial cannulation are presented in table 3.

Postoperative complications and hospital events of patients who did and did not develop brachial arterial complications are presented in table 4 . The incidence of cardiac arrest, requirement for extracorporeal membrane oxygenation support, use of intraaortic balloon counterpulsation, kidney failure, requirement for dialysis, prolonged ventilation, and multisystem organ failure was between 5 and 20 times greater in patients who had complications related to brachial arterial catheterization than in those who did not. Tertiary results on associations between brachial arterial catheter complication and length of hospitalization and inhospital mortality using multivariate regression are shown in table 5. Patients with complications had median hospital stays of 22 days compared with 8 days for patients without complications. In-hospital mortality was $24 \%$ in patients with brachial arterial complications compared with $1.75 \%$ in patients without arterial complications. 
Table 1. Summary of Patient Demographics, Baseline, and Procedural Characteristics $(N=21,597)$

\begin{tabular}{|c|c|c|c|c|}
\hline \multirow[b]{2}{*}{ Variables } & \multicolumn{2}{|c|}{$\begin{array}{l}\text { Definitive or Possible Brachial } \\
\text { Artery Cannulation Complication } \\
\qquad(\mathrm{N}=41)\end{array}$} & \multicolumn{2}{|c|}{$\begin{array}{l}\text { No Brachial } \\
\text { Artery Cannulation Complication } \\
\qquad(\mathrm{N}=21,556)\end{array}$} \\
\hline & $\begin{array}{c}\text { No. } \\
\text { Missing* }\end{array}$ & $\begin{array}{l}\text { Summary } \\
\text { Statistics }\end{array}$ & $\begin{array}{l}\text { No. } \\
\text { Missing }\end{array}$ & $\begin{array}{l}\text { Summary } \\
\text { Statistics }\end{array}$ \\
\hline Age, yr & & $64 \pm 16$ & & $64 \pm 14$ \\
\hline Sex (male), n (\%) & & $17(41)$ & & $14,317(66)$ \\
\hline Race, n (\%) & 1 & & 373 & \\
\hline White & & $30(75)$ & & $19,169(90)$ \\
\hline African American & & $6(15)$ & & $1,232(6)$ \\
\hline Others & & $4(10)$ & & $782(4)$ \\
\hline Body mass index, $\mathrm{kg} / \mathrm{m}^{2}$ & & $27.6[23.4,31.9]$ & 8 & $27.7[24.6,31.6]$ \\
\hline \multicolumn{5}{|l|}{ Medical history, n (\%) } \\
\hline Coronary artery disease & & $24(59)$ & & $9,152(42)$ \\
\hline Myocardial infarction & & $9(22)$ & & $4,071(19)$ \\
\hline Heart failure & & $17(41)$ & & $5,361(25)$ \\
\hline Hypertension & & $28(68)$ & & $14,750(68)$ \\
\hline Stroke or cerebral vascular accident & & $2(5)$ & & $1,876(9)$ \\
\hline Diabetes mellitus & & $15(37)$ & & $5,447(25)$ \\
\hline Carotid disease & & $0(0)$ & & $202(1)$ \\
\hline Peripheral arterial disease & & $14(34)$ & & $2,391(11)$ \\
\hline Chronic obstructive pulmonary disease & & $17(41)$ & & $4,405(20)$ \\
\hline Endocarditis & & $6(15)$ & & $1,254(6)$ \\
\hline Chronic kidney disease $\dagger$ & & $7(17)$ & 27 & $890(4)$ \\
\hline Dialysis & & $3(7)$ & & $342(2)$ \\
\hline Atrial fibrillation/flutter & 17 & $10(42)$ & 8,665 & $3,479(27)$ \\
\hline Complete heart block/pacer & 17 & $0(0)$ & 8,668 & $566(4)$ \\
\hline Ventricular arrhythmia & 17 & $5(21)$ & 8,668 & $958(7)$ \\
\hline Smoking & & $22(54)$ & & $9,881(46)$ \\
\hline ASA physical status, $\mathrm{n}(\%)$ & & & 1 & \\
\hline 1 & & $0(0)$ & & $1(0)$ \\
\hline 2 & & $0(0)$ & & $50(0)$ \\
\hline 3 & & $2(5)$ & & $3,570(17)$ \\
\hline 4 & & $39(95)$ & & $17,678(82)$ \\
\hline 5 & & $0(0)$ & & $254(1)$ \\
\hline 6 & & $0(0)$ & & $2(0)$ \\
\hline New York Heart Association Functional Class, n (\%) & 4 & & 3,044 & \\
\hline 1 & & $6(16)$ & & $5,020(27)$ \\
\hline 2 & & $13(35)$ & & $7,602(41)$ \\
\hline 3 & & $13(35)$ & & $4,674(25)$ \\
\hline 4 & & $5(14)$ & & $1,216(7)$ \\
\hline \multicolumn{5}{|l|}{ Preoperative laboratory values } \\
\hline Blood urea nitrogen, mg/dl & 0 & $18[14,27]$ & 27 & $18[15,23]$ \\
\hline Bilirubin, mg/dl & 1 & $0.5[0.4,0.6]$ & 213 & $0.6[0.4,0.8]$ \\
\hline Creatinine, mg/dl & & $1.0[0.9,1.2]$ & 27 & $1.0[0.8,1.2]$ \\
\hline Hematocrit, \% & & $37[31,39]$ & 4 & $39[34,42]$ \\
\hline HDL cholesterol, mg/dl & 15 & $44[37,51]$ & 4,633 & $46[37,58]$ \\
\hline LDL cholesterol, mg/dl & 15 & $81[62,104]$ & 4,710 & $88[67,114]$ \\
\hline Triglycerides, mg/dl & 15 & $101[78,114]$ & 4,632 & $101[73,144]$ \\
\hline \multicolumn{5}{|l|}{ Surgical procedure, n (\%) } \\
\hline Coronary artery bypass grafting & & $21(51)$ & & $7,145(33)$ \\
\hline Aortic valve replacement & & $16(39)$ & & $7,947(37)$ \\
\hline Aortic valve repair & & $2(5)$ & & $1,068(5)$ \\
\hline Mitral valve replacement & & $7(17)$ & & $2,340(11)$ \\
\hline Mitral valve repair & & $9(22)$ & & $5,172(24)$ \\
\hline Pulmonary valve repair/replacement & & $0(0)$ & & $133(2)$ \\
\hline Tricuspid valve replacement & & $0(0)$ & & $130(1)$ \\
\hline
\end{tabular}

(Continued) 


\begin{tabular}{|c|c|c|c|c|}
\hline \multirow[b]{2}{*}{ Variables } & \multicolumn{2}{|c|}{$\begin{array}{l}\text { Definitive or Possible Brachial } \\
\text { Artery Cannulation Complication } \\
\qquad(\mathrm{N}=41)\end{array}$} & \multicolumn{2}{|c|}{$\begin{array}{c}\text { No Brachial } \\
\text { Artery Cannulation Complication } \\
(\mathrm{N}=21,556)\end{array}$} \\
\hline & $\begin{array}{l}\text { No. } \\
\text { Missing* }\end{array}$ & $\begin{array}{l}\text { Summary } \\
\text { Statistics }\end{array}$ & $\begin{array}{l}\text { No. } \\
\text { Missing* }\end{array}$ & $\begin{array}{l}\text { Summary } \\
\text { Statistics }\end{array}$ \\
\hline Tricuspid valve repair & & $7(17)$ & & $2,153(10)$ \\
\hline Maze procedure & & $5(12)$ & & $2,680(12)$ \\
\hline $\begin{array}{l}\text { Any aortic root, ascending aortic, or aortic arch } \\
\text { replacement procedure }\end{array}$ & & $12(29)$ & & $3,510(16)$ \\
\hline Descending aorta replacement & & $2(5)$ & & $393(2)$ \\
\hline Congenital ASD/PFO suture closure & & $1(2)$ & & $372(2)$ \\
\hline Removal of atrial myxoma, cardiac tumors & & $0(0)$ & & $108(1)$ \\
\hline Carotid endarterectomy & & $0(0)$ & & $50(0)$ \\
\hline Pulmonary endarterectomy & & $1(2)$ & & $88(0)$ \\
\hline Any congenital heart disease procedure & & $1(2)$ & & $632(3)$ \\
\hline Left ventricular reconstructive procedure & & $1(2)$ & & $116(1)$ \\
\hline Pericardiectomy & & $1(2)$ & & $142(1)$ \\
\hline Septal myectomy & & $0(0)$ & & $1,157(5)$ \\
\hline Heart transplant & & $1(2)$ & & $260(1)$ \\
\hline Insertion of mechanical ventricular assist device & & $14(34)$ & & $1,032(5)$ \\
\hline \multicolumn{5}{|l|}{ History of cardiothoracic surgical procedures, $n$ (\%) } \\
\hline First surgery & & $26(64)$ & & $16,292(76)$ \\
\hline First reoperative surgery & & $12(29)$ & & $4,103(19)$ \\
\hline Second or more reoperative surgery & & $3(7)$ & & $1,161(5)$ \\
\hline Emergent surgery, n (\%) & & $3(7)$ & 1 & $816(4)$ \\
\hline \multicolumn{5}{|l|}{ Intraoperative } \\
\hline Duration of surgery, min & & $494[376,557]$ & & $359[298,441]$ \\
\hline Cardiopulmonary bypass time, min & & $129[98,182]$ & & $95[69,128]$ \\
\hline Aortic cross-clamp time, min & & $87[58,107]$ & & $71[50,96]$ \\
\hline Circulatory arrest, n (\%) & & $6(15)$ & & $1,125(5)$ \\
\hline Duration of circulatory arrest, min & & $41 \pm 20$ & & $26 \pm 21$ \\
\hline
\end{tabular}

Values are given as mean $\pm \mathrm{SD}$, median [first, third quartile], or $\mathrm{n}(\%)$, as appropriate.

*The data that were not collected in the medical records marked as missing, which is mostly race, preoperative laboratory results, New York Heart Association Functional Class, and preoperative EKG. †Chronic kidney disease was identified if eGFR was less than $30 \mathrm{ml}^{\circ} \mathrm{min}^{-1} \cdot 1.73 \mathrm{~m}^{-2}$; eGFR was calculated based on the MDRD equation.

$\mathrm{ASA}=$ American Society of Anesthesiologists; $\mathrm{ASD}=$ atrial septal defect; eGFR = estimated glomerular filtration rate; $\mathrm{EKG}=\mathrm{electrocardiogram}$; HDL = highdensity lipoprotein; LDL = low-density lipoprotein; MDRD = Modification of Diet in Renal Disease; PFO = patent foramen ovale.

\section{Discussion}

In more than 21,500 patients who had brachial arterial catheterization for cardiac surgery, complications were infrequent, occurring in less than $0.2 \%$ of patients. This low incidence of complications is comparable with that reported for radial, femoral, and brachial arterial line cannulation in cardiac surgical, noncardiac surgical, and critically ill patients. $3,7,8,10$ None of these other reports, however, included an adequate number of brachial arterial catheters to accurately assess the incidence of catheter-related complications of the brachial artery. $3,7,8$

Hypotension is associated with acute kidney and myocardial injury in (noncardiac) surgical patients, ${ }^{5}$ and accurate measurement of arterial blood pressure may improve clinical outcomes. Radial arterial systolic pressure is higher than central aortic pressure because amplification occurs as distance from the heart increases, ${ }^{11}$ although diastolic and mean arterial pressures decrease only slightly (about 1 to $4 \mathrm{mmHg}$ ). ${ }^{11-13}$ Furthermore, radial artery pressure may underestimate central aortic pressure during and immediately after cardiopulmonary bypass ${ }^{3,6,14}$ due in part to arterial spasm or thermoregulatory vasomotion. ${ }^{14-17}$ If radial arterial pressure is inaccurate, patient care may be compromised by promoting administration of vasopressor or inotropic drugs, which may themselves worsen outcomes. ${ }^{18}$ In patients having cardiac surgery, brachial arterial pressure measurements better estimate mean and systolic central aortic pressures than radial arterial pressures, ${ }^{3,19}$ and those who require hypothermic circulatory arrest are at especially high risk for developing large and prolonged radial-to-aorta pressure gradients. ${ }^{20}$ Use of the brachial artery for intraarterial pressure monitoring may thus provide a more accurate tool for hemodynamic monitoring.

The brachial artery is the main blood supply to the forearm and hand. Before the brachial artery bifurcates into the radial and ulnar arteries, several branches, including the deep brachial, humeral nutrient, superior and inferior 
Table 2. Incidence of Definitely or Possibly Related Complications from Brachial Arterial Cannulation: Primary Results $(\mathrm{N}=21,597)$

\begin{tabular}{lcc}
\hline & $\begin{array}{c}\text { Cases, } \\
\text { No. }\end{array}$ & Incidence $(\mathrm{Cl})^{*}$ \\
\hline $\begin{array}{l}\text { Definitely or possibly related } \\
\text { brachial arterial complica- }\end{array}$ & 41 & $0.19 \%(0.14-0.26)$ \\
tions $\dagger$ & & \\
Brachial artery vascular & 33 & $0.15 \%(0.10-0.23)$ \\
$\quad$ complications & 21 & \\
$\quad$ Upper extremity ischemia & 17 & \\
Thrombosis & 2 & \\
Embolism & 4 & \\
Aneurysm & 1 & \\
$\quad$ Compartment syndrome, & & \\
$\quad$ forearm & 1 & \\
$\quad$ Other arterial injury & 8 & $0.04 \%(0.02-0.08)$ \\
Infection $\neq$ & 2 & \\
$\quad$ Catheter-site infection & 1 & \\
$\quad$ Cellulitis & 7 & \\
Bloodstream infection/ & \\
$\quad$ septicemia & 0 & $0.00 \%(0.00-0.03)$ \\
Neurologic complications &
\end{tabular}

*We reported $95 \%$ Cls for the estimate of the incidence of brachial arterial complications; for the specific complications, we reported $98.3 \% \mathrm{Cl}$, which is Bonferroni adjusted $(0.05 / 3=0.017)$ for multiple outcomes. †A patient might have more than one of the conditions in this list. $f$ Five of eight cases of infection were line sepsis. These were identified as complications possibly related to brachial arterial cannulation, because it was not possible to determine whether the arterial catheter or a coexisting centra venous catheter was the source of infection.

ulnar collateral artery, provide collateral blood flow to the elbow and upper arm. However, forearm and hand perfusion via these collaterals usually is insufficient if acute occlusion of the brachial artery occurs. Thus vascular compromise can cause ischemia to the arm and consequent catastrophic injury, and is an overwhelming concern of brachial arterial cannulation. As might be expected, nearly $80 \%$ of all the complications were related to vascular injury or occlusion.
The overall incidence of vascular complications, however, was low at $0.15 \%$ (i.e., less than 1 per 650 catheters).

Arterial catheters were removed immediately when complications were detected. Patients with brachial artery thrombosis received either thrombectomy or open repair of the brachial artery and postoperative anticoagulation. The remaining patients with upper extremity ischemia improved with intravenous anticoagulation. Ultrasoundguided compression was used to treat brachial arterial pseudoaneurysms. ${ }^{21}$ With one exception, ${ }^{7}$ other studies similarly reported that surgical and/or medical treatment was required for all vascular complications. ${ }^{3,8,10}$

Handlogten $e t$ al. ${ }^{8}$ reported a slightly greater rate of complications compared with our results after brachial arterial catheterization, namely $0.35 \%$; however, this estimate was based on 3 complications among 858 patients, a fragile result that would be altered substantially by the addition or subtraction of a single complication. Nuttall et al. ${ }^{7}$ similarly reported a complication rate of $0.75 \%$ in cardiac surgical patients, which was also based on a single complication in 134 patients; however, their overall complication rate of $0.12 \%$ in approximately 1,600 patients was comparable with our report.

Although vascular complications after radial arterial cannulation recently were reported to be approximately $0.03 \%$ overall, complications were higher in patients undergoing cardiac surgery (approximately $0.07 \%) .^{7}$ Though our vascular complication rate in cardiac surgical patients of $0.15 \%$ was slightly greater, ${ }^{7}$ the $95 \%$ CIs likely overlap. A direct comparison of safety between radial versus brachial arterial lines is not possible by comparing the results of separate reports because of confounding by differences in patient populations and risk factors. ${ }^{7}$ Nonetheless, complications after brachial arterial cannulation may be slightly higher by perhaps 7 to 8 complications per 10,000 brachial catheters compared with radial arterial cannulation. The incidence

Table 3. The Association of Patient Comorbidities with Brachial Arterial Complications: Secondary Results $(N=21,597)$

\begin{tabular}{|c|c|c|c|c|}
\hline \multirow[b]{2}{*}{ Baseline Comorbidities } & \multicolumn{2}{|c|}{ Univariate Association } & \multicolumn{2}{|c|}{ Multivariate Association $†$} \\
\hline & OR $(99 \% \mathrm{Cl})^{*}$ & $P$ Value $^{*}$ & $\begin{array}{l}\text { Adjusted OR } \\
(99 \% \mathrm{Cl})^{\star}\end{array}$ & $P$ Value* \\
\hline Diabetes & $1.71(0.74-3.94)$ & 0.10 & $1.17(0.45-3.03)$ & 0.68 \\
\hline Peripheral arterial disease & $4.16(1.78-9.73)$ & $<0.001$ & $2.78(1.11-7.01)$ & 0.004 \\
\hline Carotid disease $\ddagger$ & N/A & N/A & N/A & N/A \\
\hline Coronary artery disease & $1.91(0.84-4.33)$ & 0.04 & $1.46(0.59-3.59)$ & 0.28 \\
\hline Chronic kidney disease§ & $4.78(1.69-13.51)$ & $<0.001$ & $2.31(0.70-7.65)$ & 0.07 \\
\hline
\end{tabular}

${ }^{*}$ The significance criteria for all five secondary tests were 0.01 , which is Bonferroni adjusted for multiple comparisons $(0.05 / 5=0.01)$ and corresponds to $99 \%$ Cls. †The association between the baseline condition (history of diabetes, peripheral vascular disease, carotid disease, coronary artery disease, chronic kidney disease) and postoperative brachial artery cannulation complication outcome was adjusted for patient's age, body mass index, sex, race, ASA status, duration of surgery, surgery emergency status, number of simultaneous cardiac procedures, duration of cardiopulmonary bypass time, duration of myocardial ischemia, and duration of circulatory arrest. $\neq$ Carotid artery disease was identified if Doppler evaluation demonstrated $\geq 50 \%$ stenosis. There were no patients with carotid disease among 41 patients who experienced complications related to brachial artery cannulation. Therefore, we were not able to report the association between presence of carotid disease and risk of brachial artery cannulation complication. §Chronic kidney disease was identified if eGFR was less than $30 \mathrm{ml} \cdot \mathrm{min}^{-1} \cdot 1.73 \mathrm{~m}^{-2}$; eGFR was calculated based on the MDRD equation.

ASA = American Society of Anesthesiologists; eGFR = estimate glomerular filtration rate; MDRD = Modification of Diet in Renal Disease; N/A = not applicable; OR = odds ratio. 
Table 4. Postoperative Complications and Hospital Events in Patients with and without Brachial Arterial Cannulation Complication

\begin{tabular}{lcc}
\hline & $\begin{array}{c}\text { Definitive or Possible Brachial Artery } \\
\text { Cannulation Complication }\end{array}$ & $\begin{array}{c}\text { No Brachial Artery Cannulation } \\
\text { Complication }\end{array}$ \\
\cline { 2 - 3 } Postoperative Complications & $(\mathrm{N}=41)$ & $(\mathrm{N}=21,556)$ \\
\hline Cardiac arrest & $5(12)$ & $390(2)$ \\
Atrial fibrillation & $17(41)$ & $6,081(28)$ \\
Extracorporeal membrane oxygenation & $7(17)$ & $214(1)$ \\
Left ventricular assist device & $1(2)$ & $306(1)$ \\
Intra/postoperative intraaortic balloon counterpulsation & $6(15)$ & $527(2)$ \\
Stroke & $4(10)$ & $291(1)$ \\
Transient ischemic attack & $0(0)$ & $32(0)$ \\
Coma/encephalopathy & $1(2)$ & $220(1)$ \\
Renal failure & $13(32)$ & $1,018(5)$ \\
Renal failure requiring dialysis & $8(20)$ & $469(2)$ \\
Prolonged ventilation: $>$ 24h postoperatively & $30(73)$ & $3,344(16)$ \\
Pneumonia & $4(10)$ & $273(1)$ \\
Septicemia or bacteremia or sepsis & $11(27)$ & $224(1)$ \\
Gastrointestinal complication & $3(7)$ & $767(4)$ \\
Multisystem organ failure & $4(10)$ & $87(0)$ \\
\hline
\end{tabular}

Data are shown as $\mathrm{n}(\%)$.

Table 5. The Association between Brachial Arterial Cannulation Complications and Length of Hospital Stay and In-hospital Mortality: Tertiary Results

\begin{tabular}{|c|c|c|c|c|c|c|}
\hline \multirow[b]{2}{*}{ Outcomes } & \multicolumn{2}{|c|}{ Raw Data } & \multicolumn{2}{|c|}{ Univariate Association } & \multicolumn{2}{|c|}{ Multivariate Association* } \\
\hline & $\begin{array}{l}\text { Definitive or Possible } \\
\text { Brachial Artery Can- } \\
\text { nulation Complication }\end{array}$ & $\begin{array}{c}\text { No Brachial Artery } \\
\text { Cannulation } \\
\text { Complication }\end{array}$ & $\begin{array}{l}\text { Estimate } \\
(97.5 \% \mathrm{Cl})\end{array}$ & $P$ Value $†$ & $\begin{array}{l}\text { Estimate } \\
(97.5 \% \mathrm{Cl})\end{array}$ & $P$ Value $†$ \\
\hline & $(\mathrm{n}=41)$ & $(n=21,556)$ & & & & \\
\hline $\begin{array}{l}\text { Length of hospi- } \\
\text { talization, } d\end{array}$ & $22[14,30]$ & $8[6,14]$ & $2.29(1.82-2.87) \ddagger$ & $<0.001$ & $1.55(1.28-1.89) \ddagger$ & $<0.001$ \\
\hline $\begin{array}{l}\text { In-hospital } \\
\text { mortality }\end{array}$ & $10(24 \%)$ & $378(1.75 \%)$ & $18.07(7.93-41.18) \S$ & $<0.001$ & 8.19 (3.08-21.78)§ & $<0.001$ \\
\hline
\end{tabular}

The raw data are reported as median [first, third quartiles] or $\mathrm{n}(\%)$, as appropriate $(\mathrm{N}=21,597)$.

*Multivariate linear regression was used to assess the ratio of geometric means of length of hospitalization comparing patients with brachial artery complication with those without complication along with $97.5 \% \mathrm{Cl}$. Multivariate logistic regression was used to assess the odds ratio of in-hospital mortality comparing patients with brachial artery complication with those without complication. Both associations were adjusted for patient's age, body mass index, sex, race, ASA status, history of diabetes, peripheral vascular disease, carotid disease, coronary artery disease, duration of surgery, surgery emergency status, number of simultaneous cardiac procedures, duration of cardiopulmonary bypass time, duration of myocardial ischemia, and duration of circulatory arrest. †The Wald test $P$-value was reported; significance criteria were Bonferroni corrected for multiple testing and set to $0.05 / 2=0.025$. $\ddagger$ The ratio of geometric means along with $97.5 \% \mathrm{Cl}$ was reported. §The odds ratio along with $97.5 \% \mathrm{Cl}$ was reported.

ASA = American Society of Anesthesiologists.

of complications, however, may be higher at institutions in which brachial arterial cannulation is not routinely performed.

Patients having cardiac surgery may be at greater risk for complications from brachial arterial catheterization because they often have underlying vascular disease and experience perioperative hypoperfusion and low cardiac output state. Use of inotropic and vasopressor therapy may further jeopardize peripheral tissue perfusion. Alternatively, anticoagulation during cardiopulmonary bypass, perioperative platelet dysfunction or coagulopathy, and hypothermia impair coagulation $^{22}$ and might reduce thrombotic complications. Therefore, the extent to which our observed incidence in cardiac surgical patients can be extrapolated to patients having other types of surgery remains unknown. Although Nuttall et al. ${ }^{7}$ reported more complications from brachial arterial cannulation for cardiac than noncardiac surgery, only a small number of patients were included, leaving the issue essentially unresolved.

Only 3 of 21,597 patients $(0.01 \%)$ developed infectious complications definitely related to brachial arterial catheterization. Five additional patients developed catheter-related bloodstream infections, but definitive evidence that the arterial catheter was the source of infection was absent. Presumably, coexisting central venous catheters were the more likely source of infection. ${ }^{23}$ We nonetheless conservatively included these five patients as possible infectious complications. Our incidence of infectious complications is less than 
most reports, which range from 0.02 to $0.36 \%, 7,24$ depending on site of arterial cannulation and patient population. Patients with infectious complications were treated with broad-spectrum intravenous antibiotics, whereas nearly one half required vasopressors infusion due to septic shock.

The median nerve runs close to the brachial artery in the antecubital fossa and paresthesia of the median nerve are common during brachial arterial cannulation. We thus expected some nerve injuries; however, we did not observe any reported nerve injury plausibly related to brachial artery cannulation among 21,597 patients. Previous studies also report no nerve injuries, although none were even remotely powered for this rare complication. ${ }^{3,7,8}$ Injury to the median nerve during brachial arterial cannulation is thus exceedingly rare and does not cause persistent neurologic dysfunction. Fear of this complication should not guide selection of arterial cannulation site.

Unsurprisingly, patients with peripheral arterial disease had increased risk of complications from brachial arterial cannulation, possibly related to the presence of atheroma formation, calcification of the peripheral arteries, luminal narrowing, and reduced blood flow. ${ }^{25}$ We note, however, that the overall incidence of complications even in these patients was low and that peripheral arterial disease is hardly a compelling reason to avoid brachial arterial catheterization if otherwise indicated. However, as mentioned previously, our low incidence of complications in cardiac surgical patients cannot be directly extrapolated to patients with peripheral vascular disease having noncardiac surgery, because perioperative management, such as systemic anticoagulation during cardiac surgery, may reduce risk of complications. Interestingly, patients with diabetes mellitus, coronary artery disease, and chronic kidney disease, all of which are associated with arterial compromise, were not at increased risk from brachial arterial cannulation. Safety of brachial arterial cannulation has significant future implications, especially in patients with kidney disease, who may later require vascular access for hemodialysis. We examined risk in patients with carotid disease, since this finding may indicate disease in other major arteries. However, none of the patients with brachial arterial line complications had preexisting carotid disease, suggesting that patients with carotid artery disease are not at increased risk, although we could not formally assess the association.

Patients with arterial line complications had prolonged hospital stays and greater in-hospital mortality. The postoperative course in these patients, however, was complicated by a much greater incidence of severe life-threatening postoperative complications, including cardiac arrest, kidney injury requiring dialysis, multiorgan failure, and use of extracorporeal membrane oxygenation for severe cardiac or pulmonary failure refractory to conventional management. It seems obvious that these serious postoperative complications were not directly consequent to brachial arterial cannulation. Instead, it is more likely that prolonged cannulation and monitoring of the brachial artery was required in these critically ill patients who suffered from a low cardiac output state, required high-dose vasopressor therapy or mechanical circulatory support, or had compromised systemic and peripheral perfusion. Thus, these serious postoperative complications likely predisposed patients to brachial arterial line complications, rather than the possibility that brachial arterial cannulation caused life-threatening complications, a prolonged hospital stay, or greater in-hospital mortality. Although the overall incidence of complications is low, increased clinical suspicion for vascular complications and appropriate consideration for alternative arterial line sites are suggested for critically ill patients who require prolonged high-dose pharmacologic or mechanical circulatory support.

Our observational analysis has the limitations inherent to its retrospective design. As in all retrospective studies, our findings rely on accurate recording of arterial line catheterization, comorbidities, and postoperative complications, and thus there is the possibility of underreporting of complications. We were not able to report minor complications due to the lack of in-hospital International Classification of Diseases, Ninth Revision and Current Procedural Terminology billing codes for less severe complications. It is thus likely that we missed minor selflimited complications, but such complications are also of questionable clinical importance. Unlike one investigation that reported minor clinically insignificant complications, such as "temporary arterial occlusion," ${ }^{24}$ we considered only clinically important vascular complications which included upper extremity ischemia, arterial thrombosis, pseudoaneurysm, and compartment syndrome. Our investigation could not compare the risk of complications between radial and brachial arterial lines, because radial arterial lines are only used at our institution when contraindications or difficulty with brachial arterial cannulation are present.

Although our secondary analysis adjusted for multiple confounding variables, the possibility of unmeasured covariates and residual confounders cannot be excluded. We were unable to determine the true incidence of infection because catheter tips were not always cultured; however, we conservatively included patients who experienced line-related sepsis, even though the central venous catheter was the more likely source of infection. Arterial line documentation was missing in approximately $20 \%$ of patients, mostly during the new implementation of our intraoperative electronic documentation record when documentation was in development. However, it seems unlikely that including these patients would substantively influence our conclusions.

In summary, brachial artery cannulation during cardiac surgery was associated rarely with complications. Most complications, unsurprisingly, were vascular. Because brachial artery pressure measurements better reflect aortic pressure 
than radial arterial catheters and complications are rare, the brachial artery remains a reasonable site for direct arterial pressure measurement.

\section{Research Support}

This investigation was funded by the Departments of Cardiothoracic Anesthesia and Outcomes Research at the Cleveland Clinic, Cleveland, Ohio.

\section{Competing Interests}

The authors declare no competing interests.

\section{Correspondence}

Address correspondence to Dr. Duncan: Departments of Cardiothoracic Anesthesiology and Outcomes Research, Cleveland Clinic 9500 Euclid Avenue / J4-331, Cleveland, Ohio 44195. duncana@ccf.org. Information on purchasing reprints may be found at www.anesthesiology.org or on the masthead page at the beginning of this issue. Anesthesiology's articles are made freely accessible to all readers, for personal use only, 6 months from the cover date of the issue.

\section{References}

1. Brzezinski M, Luisetti T, London MJ: Radial artery cannulation: a comprehensive review of recent anatomic and physiologic investigations. Anesth Analg 2009; 109:1763-81

2. Dorman T, Breslow MJ, Lipsett PA, Rosenberg JM, Balser JR, Almog Y, Rosenfeld BA: Radial artery pressure monitoring underestimates central arterial pressure during vasopressor therapy in critically ill surgical patients. Crit Care Med 1998; 26:1646-9

3. Bazaral MG, Welch M, Golding LA, Badhwar K: Comparison of brachial and radial arterial pressure monitoring in patients undergoing coronary artery bypass surgery. ANESTHESIOLOGY $1990 ; 73: 38-45$

4. Fuda G, Denault A, Deschamps A, Bouchard D, Fortier A, Lambert J, Couture P: Risk factors involved in centralto-radial arterial pressure gradient during cardiac surgery. Anesth Analg 2016; 122:624-32

5. Mascha EJ, Yang D, Weiss S, Sessler DI: Intraoperative mean arterial pressure variability and 30-day mortality in patients having noncardiac surgery. ANESTHESIOLOGY 2015; 123:79-91

6. Gravlee GP, Wong AB, Adkins TG, Case LD, Pauca AL: A comparison of radial, brachial, and aortic pressures after cardiopulmonary bypass. J Cardiothorac Anesth 1989; 3:20-6

7. Nuttall G, Burckhardt J, Hadley A, Kane S, Kor D, Marienau MS, Schroeder DR, Handlogten K, Wilson G, Oliver WC: Surgical and patient risk factors for severe arterial line complications in adults. ANESTHESIOLOGY 2016; 124:590-7

8. Handlogten KS, Wilson GA, Clifford L, Nuttall GA, Kor DJ: Brachial artery catheterization: an assessment of use patterns and associated complications. Anesth Analg 2014; 118:288-95

9. Brown LD, Cai TT, DasGupta A: Interval estimation for a binomial proportion. Stat Sci 2001;16:101-17
10. Mann S, Jones RI, Millar-Craig MW, Wood C, Gould BA, Raftery EB: The safety of ambulatory intra-arterial pressure monitoring: a clinical audit of 1000 studies. Int J Cardiol 1984; 5:585-97

11. McEniery CM, Cockcroft JR, Roman MJ, Franklin SS, Wilkinson IB: Central blood pressure: current evidence and clinical importance. Eur Heart J 2014; 35:1719-25

12. Safar ME, Plante GE, Mimran A: Arterial stiffness, pulse pressure, and the kidney. Am J Hypertens 2015; 28:561-9

13. O'Rourke MF, Seward JB: Central arterial pressure and arterial pressure pulse: new views entering the second century after Korotkov. Mayo Clin Proc 2006; 81:1057-68

14. Baba T, Goto T, Yoshitake A, Shibata Y: Radial artery diameter decreases with increased femoral to radial arterial pressure gradient during cardiopulmonary bypass. Anesth Analg 1997; 85:252-8

15. Stern DH, Gerson JI, Allen FB, Parker FB: Can we trust the direct radial artery pressure immediately following cardiopulmonary bypass? ANESTHESIOLOGY 1985; 62:557-61

16. Kanazawa M, Fukuyama H, Kinefuchi Y, Takiguchi M, Suzuki T: Relationship between aortic-to-radial arterial pressure gradient after cardiopulmonary bypass and changes in arterial elasticity. Anesthesiology 2003; 99:48-53

17. Urzua J, Sessler DI, Meneses G, Sacco CM, Canessa R, Lema G: Thermoregulatory vasoconstriction increases the difference between femoral and radial arterial pressures. J Clin Monit 1994; 10:229-36

18. Nielson E, Hennrikus E, Lehman E, Mets B: Angiotensin axis blockade, hypotension, and acute kidney injury in elective major orthopedic surgery. J Hosp Med 2014; 9:283-8

19. De Hert SG, Vermeyen KM, Moens MM, Hoffmann VL, Bataillie KJ: Central-to-peripheral arterial pressure gradient during cardiopulmonary bypass: relation to pre- and intra-operative data and effects of vasoactive agents. Acta Anaesthesiol Scand 1994; 38:479-85

20. Manecke GR Jr, Parimucha M, Stratmann G, Wilson WC, Roth DM, Auger WR, Kerr KM, Jamieson SW, Kapelanski DP, Mitchell MM: Deep hypothermic circulatory arrest and the femoral-to-radial arterial pressure gradient. J Cardiothorac Vasc Anesth 2004; 18:175-9

21. Luedde M, Krumsdorf U, Zehelein J, Ivandic B, Dengler T, Katus HA, Tiefenbacher C: Treatment of iatrogenic femoral pseudoaneurysm by ultrasound-guided compression therapy and thrombin injection. Angiology 2007; 58:435-9

22. Polderman KH: Mechanisms of action, physiological effects, and complications of hypothermia. Crit Care Med 2009; 37(7 Suppl):S186-202

23. Koh DB, Gowardman JR, Rickard CM, Robertson IK, Brown A: Prospective study of peripheral arterial catheter infection and comparison with concurrently sited central venous catheters. Crit Care Med 2008; 36:397-402

24. Scheer B, Perel A, Pfeiffer UJ: Clinical review: complications and risk factors of peripheral arterial catheters used for haemodynamic monitoring in anaesthesia and intensive care medicine. Crit Care 2002; 6:199-204

25. Faxon DP, Fuster V, Libby P, Beckman JA, Hiatt WR, Thompson RW, Topper JN, Annex BH, Rundback JH, Fabunmi RP, Robertson RM, Loscalzo J: Atherosclerotic Vascular Disease Conference: Writing Group III: pathophysiology. Circulation 2004;109:2617-25 
Appendix 1. Variables Used to Identify Possible Complications Related to Brachial Arterial Catheterization from (1) ICD-9 Diagnoses and Procedures Codes, (2) CPT Procedure Codes from the Cleveland Clinic PHDS Registry, and (3) Postoperative Complications from the Institutional STS Database

Complications

Description

PHDS

Vascular injury

Nerve injury

Loss of function

Infection

Institutional

Infection

Neuroparalysis

Postoperative anticoagulation

Acute limb ischemia

Noncardiac reoperations

Aneurysm of artery of upper extremity

Chronic total occlusion of artery of the extremities

Arterial embolism and thrombosis of upper extremity

Fasciotomy of hand

Stricture of artery exploration otomy forearm and/or wrist

Thrombectomy of brachial artery

Carpal tunnel syndrome

Injury to median nerve

Other neuroplasty

Other finger(s) amputation status

Below elbow amputation status abase* upper or lower limb ischemia.
Air embolism as a complication of medical care not elsewhere classified

Nontraumatic compartment syndrome of upper extremity

Surgical repair/exploration of brachial artery: decompression fasciotomy with brachial artery

Fasciotomy (possible compartment syndrome) in the antecubital fossa: decompression fasci-

Median nerve damage (tingling numbness) in antecubital fossa: neuroplasty

Digit amputation for upper extremity: amputation finger or thumb

Other specified local infections of skin and subcutaneous tissue

Arm infection: infection with type "radial artery harvest site infection" or "arm"

Bacteremia: Indicate whether a recognized pathogen is cultured from 1 or more blood cultures

Sepsis: sepsis is defined as evidence of serious infection accompanied by a deleterious systemic response. In the time period of the first 48 postoperative or postprocedural hours, the diagnosis of sepsis requires the presence of an SIRS resulting from a proven infection (such as bacteremia, fungemia, or urinary tract infection). In the time period after the first 48 postoperative or postprocedural hours, sepsis may be diagnosed by the presence of an SIRS resulting from suspected or proven infection. During the first $48 \mathrm{~h}$, an SIRS may result from the stress associated with surgery and/or cardiopulmonary bypass. Thus, the clinical criteria for sepsis during this time period should be more stringent. An SIRS is present when at least two of the following criteria are present: hypo- or hyperthermia $(>38.5$ or $<36.0)$, tachycardia or bradycardia, tachypnea, leukocytosis or leukopenia, or thrombocytopenia.

Indicate whether the patient had a new postoperative permanent or transient paralysis, paraparesis, or paraplegia related to spinal cord ischemia and not related to a stroke.

Indicate whether the patient had bleeding, hemorrhage, and/or embolic events related to anticoagulant therapy postoperatively. This may include patients who experience disseminated intravascular coagulopathy or heparin-induced thrombocytopenia.

Indicate whether the patient had any complication producing limb ischemia. This may include

Indicate whether the patient returned to the operating room for other noncardiac reasons. This includes procedures requiring a return to the operating room such as tracheostomy or general surgery procedures. This does not include procedures performed outside the operating room such as gastrointestinal laboratory for PEG tube, shunts for dialysis, etc.

ICD-9 Diagnoses or Procedure Code or CPT Procedure Code

442.0

440.4

444.21

999.1

729.71

82.12

39.31

24495

25020-25025

34101

354.0

955.1

04.79

64718

V49.62

V49.65

26951, 26952

686.8

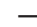

$-$

${ }^{\star}$ The description corresponds to STS Adult Cardiac Surgery Database Data Specifications, version 2.81.

CPT = Current Procedural Terminology; ICD-9 = International Classification of Diseases, Ninth Revision; PEG = polyethylene glycol; PHDS = Perioperative Health Documentation System; SIRS = systemic inflammatory response syndrome; STS = Society of Thoracic Surgeons. 


\begin{tabular}{lc}
\hline & $\begin{array}{c}\text { Cases, No. (\%) } \\
(\mathrm{n}=41)\end{array}$ \\
\hline Any treatment for brachial arterial complication & $37(100)$ \\
Brachial artery injury or occlusion* & $29(78)$ \\
Surgical repair of brachial artery & $8(22)$ \\
Thrombectomy & $18(49)$ \\
Fasciotomy of forearm & $1(3)$ \\
Anticoagulation & $17(46)$ \\
Amputation & $0(0)$ \\
Compression & $3(8)$ \\
Thrombin injection & $1(3)$ \\
Rooke ${ }^{\circledR}$ mitt & $3(8)$ \\
Other & $9(2)$ \\
Infection & $8(12)$ \\
Antibiotic therapy & $8(12)$ \\
Median nerve injury & $0(0)$ \\
\hline
\end{tabular}

${ }^{*} \mathrm{~A}$ patient might have multiple treatments.

\section{ANESTHESIOLOGY REFLECTIONS FROM THE WOOD LIBRARY-MUSEUM}

\section{Nitrous Oxide from a Peach of a Specialist: J. Warren Worcester, M.D.}

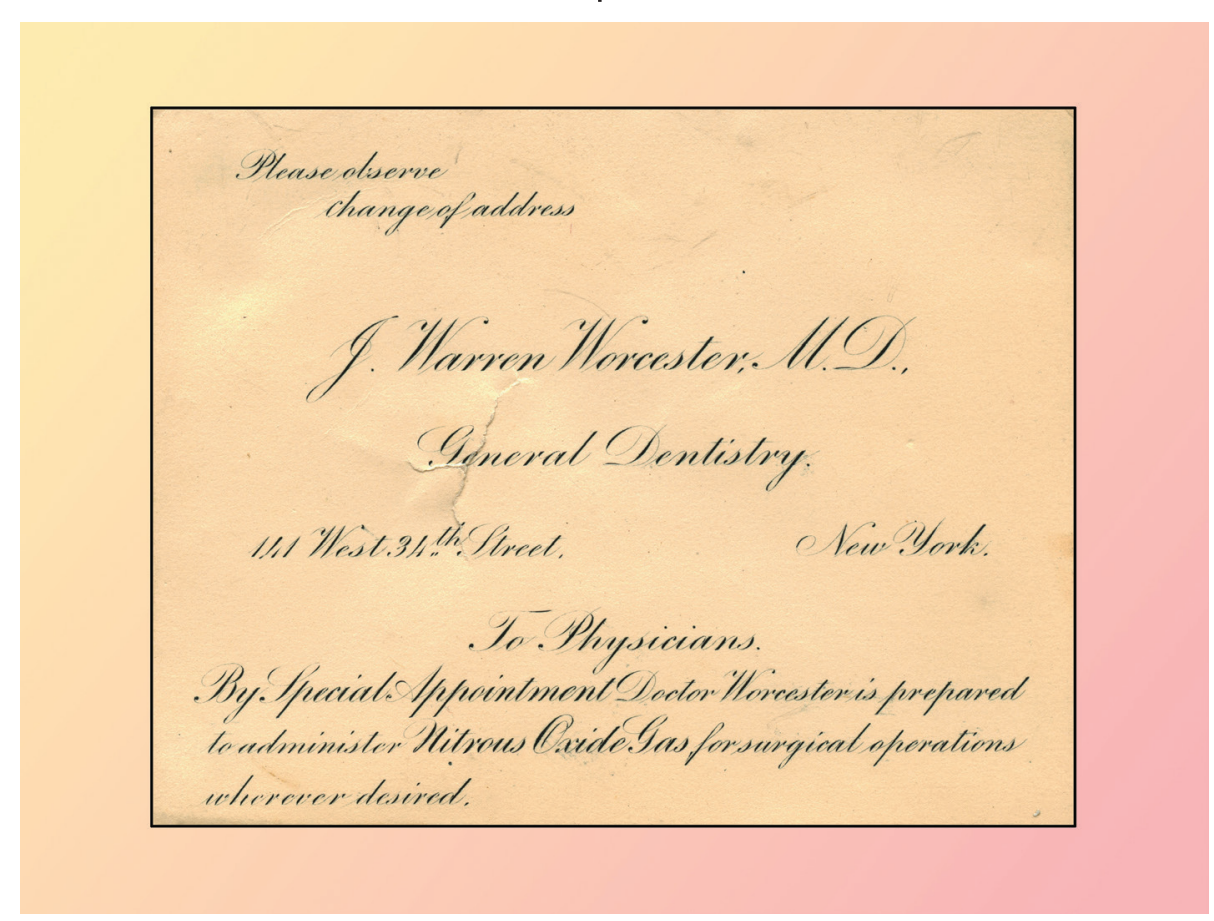

After attending lectures at the New York College of Pharmacy and then the nearby College of Physicians and Surgeons, Joseph Warren Worcester (1860 to 1926) earned his M.D. in 1888 from another College of Physicians and Surgeons, the one in Baltimore, Maryland. He returned that same year to New York to marry and live with Ella Hallock, a lifelong Middletown resident. According to this now peach-framed trade card from the Wood Library-Museum's Ben Z. Swanson Collection, Doctor Worcester was "prepared / to administer Nitrous Oxide Gas for surgical operations / wherever desired." Branding himself a "Specialist in use of nitrous oxide gas for dental and general surgery," this physician and dental surgeon cited his professional "change of address" to Manhattan's "141 West 34th Street." Using that clue, a diligent historical researcher can comb city directories and newspapers and date this card to c. 1892. By that year, this versatile physician had successfully bred the fruit brand for which he would become nationally renowned, the "Dr. Worcester Peach." (Copyright (c) the American Society of Anesthesiologists' Wood Library-Museum of Anesthesiology.)

George S. Bause, M.D., M.P.H., Honorary Curator and Laureate of the History of Anesthesia, Wood LibraryMuseum of Anesthesiology, Schaumburg, Illinois, and Clinical Associate Professor, Case Western Reserve University, Cleveland, Ohio. UJYC@aol.com. 